\title{
ANÁLISE DA DISTRIBUIÇÃO DA REGIÃO DOS FERIMENTOS PROVOCADOS POR MORCEGOS HEMATÓFAGOS DESMODUS ROTUNDUS (GEOFFROY, 1810) EM BOVINOS SOB CONDIÇÕES DE CAMPO
}

\author{
S.N.Pereira ${ }^{1}$, C.B. Gittii ${ }^{2}$, M.M.O. Cabral ${ }^{1,3}$ \\ ${ }^{1}$ Universidade "SeverinoSombra", Centro deCiências, Tecnologicas eda Natureza, Laboratório de Insetos, Av. \\ ExpedicionárioOswaldodeAlmeida Ranos,CEP27700-000,Vassouras, RJ, Brasil.E-mail:spereirabio@gmail.com
}

\section{RESUMO}

Existem diversas espécies de morcegos no Brasil, sendo somente três hematófagas. O morcego Desmodus rotundus (Geoffroy, 1810), conhecido como "morcego vampiro comum", apresenta-se amplamente distribuído por todo território brasileiro e é encontrado em maior número do que as outras espécies de morcegos hematófagos. É responsável pela transmissão de diferentes doenças a outros mamíferos, especialmente a raiva nos herbívoros. Com o objetivo de avaliar regionalmente os ferimentos causados pelo D. rotundus em bovinos sob condições de campo, foram estudadas 12 propriedades rurais no período de março de 2005 a março de 2006 no Município de Valença, RJ. De um total de 580 bovinos examinados, foram observados $101 \mathrm{com}$ ferimentos provocados por morcegos hematófagos (D. rotundus). Após análise dos resultados, pôde-se concluir que $D$. rotundus utiliza diferentes locais para se alimentar nos bovinos e as regiões corporais que apresentaram maior número de ferimentos foram a ântero e a póstero-superior. O elevado número de ferimentos encontrados, na metade superior dos bovinos (85,87\%), justifica a adoção de metodologias de controle populacional de morcegos hematófagos que utilizem o contato corporal desses morcegos com o dorso do animal.

PALAVRAS-CHAVE: Mordedura, bovinos, Desmodus rotundus.

\section{ABSTRACT}

TOPOGRAPHIC ANALYSIS OF WOUNDS CAUSED BY THE VAMPIRE BAT DESMODUS ROTUNDUS (GEOFFROY, 1810) IN CATTLE UNDER FIELD CONDITIONS. Brazil possesses a large amount of bat species, only 3 of which are haematophagous. The most abundant species, Desmodus rotundus (Geoffroy, 1810), is widely distributed throughout the Brazilian territory and it is involved in the transmission of several diseases to other animals, notably to herbivorous species, in the case of rabies. This study presents the topographical analysis of wounds caused by D. rotundus in bovines from 12 different rural properties in the county of Valença, state of Rio de Janeiro, where 101 bovines out of 580 examined in a year showed wounds caused by haematophagous bats. Analysis of the results leads to the conclusion that $D$. rotundus uses different sites to feed from the bovine's body and that the superior body region was the most frequently used. The increased number of wounds found in the bovine's superior half region $(85.87 \%)$ will justify the use of bat population control methodologies which take into consideration the bat's corporal contact with the dorsal region of bovines.

KEY WORDS: Wounds, cattle, Desmodus rotundus.

\section{INTRODUÇÃO}

Os morcegos hematófagos pertencem à classe Mammalia, ordem Chiroptera, subordem Microchiroptera, família Phyllostomidaee subfamília Desmodontinae. Segundo TADDEI (1983), existem três gêneros com uma espécie cada, são elas: Desmodus rotundus (Geoffroy, 1810), Diphyllaecaudata (Spix, 1823) e Diaemus youngii (Jentink, 1893).

A raiva dos herbívoros transmitida pelos $D$. rotundus ocorre somente na América Latina e a sua área de apresentação corresponde à distribuição

${ }^{2}$ Universidade Federal Rural do Rio de Janeiro, Instituto de Veterinária, Departamento de Epidemiologia e Saúde Pública, Seropédica, RJ, Brasil.

${ }^{3}$ Fundação Oswaldo Cruz, Laboratório de Díptera, Entomologia, Manguinhos, RJ, Brasil. 
geográfica da referida espécie hematófaga, ou seja, do norte da Argentina ao norte do México. Acreditase que, no período pré-colombiano, o morcego hematófago tinha como fonte de alimento a fauna silvestre de sangue quente e o homem nativo. A população de morcegos era, provavelmente, menor que a atual, entretanto, a introdução de espécies domésticas pelos colonizadores parece ter proporcionado um aumento populacional com consequente expansão territorial de D. rotundus (UIEDAetal., 2004). Estes animais domésticos, sem qualquer adaptação aos morcegos, tornaram-se presas fáceis passando a representar uma fonte abundante e acessível de alimento, tornando-se também as principais vítimas da transmissão do vírus da raiva, resultante do hábito alimentar do morcego $D$. rotundus, queéconsiderado uma "praga" da pecuária neotropical. A raiva transmitida pelo morcego hematófago tem impacto econômico significativo na pecuária da América tropical representada por sua letalidade e pela perda de sangue durante a espoliação sanguínea (FunDAÇão NACiOnAL de SAÚde, 1996).

UIEDA (1987) relatou que, durante a aproximação, o morcego se mostra bastante cauteloso e responde prontamente a qualquer reação da vítima. A aproximação do D. rotundus às suas presas podeser feita pelo pouso no corpo do bovino ou no chão, próximo a ele (SAZIMA, 1978). Caso ocorra qualquer sinal de perigo, ele se afasta do local até que o perigo cesse ou abandona este animal e sai à procura de outra presa (SAZIMA; UIEDA, 1980).

$\mathrm{O} D$. rotundus busca alimento em uma área de cerca de 5 a $8 \mathrm{~km}$ ao redor de seu abrigo (CRESPO et al., 1971). Em certas regiões, essa distância pode se estender para 15 a 20 km (MalagA-AlbA, 1954). Quando a presa está a uma distância muito longa, estes morcegos podem demorar mais de um dia para retornar ao seu abrigo principal, passando a(s) noite(s) em abrigo(s) secundário(s) (GREENHALL, 1963; AlENCAR et al., 1994). Ele voa geralmente a uma altura de 0,5 e 1,5 m, uma vez que suas presas são animais que repousam no chão, ao contrário das outras espécieshematófagas (Diaemus youngiie Diphyllaecaudata) que se alimentam de animais arborícolas (GREENHALL, 1963). Um fator importante na seleção das presas é a acessibilidade (UIEDA, 1982). O morcego escolhe e ataca geralmente aqueles animais de temperamento mais dócil e que dormem na periferia do grupo (TURNER, 1975). Tal fato poderia explicar porque, num rebanho, alguns animais são mais atacados que outros (SAZIMA, 1978). Eles utilizam um sistema de ecolocalização, com sons de alta frequência para detecção de obstáculos e orientação durante o voo (GreENHAll et al. 1969).

A reação dos animais à aproximação dos morcegos hematófagos geralmente ocorre quando estes pousam em seu corpo. O bovino reage, geralmente, com movimentos de cabeça, da cauda e da musculatura da pele (CRESPO et al., 1971).

GREENHALL (1970) relatou que os locais mais utilizados em equídeos, bovídeos, ovinos e caprinos são a espádua, o pescoço, a base dos chifres, a base das orelhas, o focinho, o cotovelo, as pernas, a cauda, a vulva e o ânus. Almeida et al. (2002), em trabalho realizadona região cárstica deCordisburgoeCurvelo, em Minas Gerais, relataram ataques por D. rotundus em várias partes do corpo dos bovinos, como coroa dos cascos, inserção da cauda, orelhas, focinho, dorso e região ínguino-crural e, com maior frequência, na região do pescoço. Citaram ainda que os morcegos sugam em todas as regiões corporais dos bovinos, mas preferencialmente nos membros anteriores, dorso, membros posteriores e cernelha, podendo provocar abertura de novos ferimentos quase que diariamente.

SAZIMA (1978) citou que um bovino foi visto sendo utilizado por três morcegos, simultaneamente, um na axila e os dois outros na região lombar, alimentandoseda mesma ferida. Emoutroanimal, com uma mordedura tambémnaaxila, observou-sequedois $D$. rotundus sealimentavamnestemesmo ferimentosucessivamente. Omaiornúmero deferimentos causados por morcegos hematófagos ocorre na região superior do corpo dos bovinos (GITTI, 2001). Não há rigor no retorno, em termos derepetiçãodos ferimentos, emestudos realizados em cativeiro por Piccinini et al. (1985).

Modificações nas condições ecológicas causadas pelohomem, tais como: desmatamentodesordenado, a ocupação destes territórios pela pecuária e a construção de abrigos artificiais em potencial para o $D$. rotundus, tornam acessíveis e constantes a presença deste mamífero (TADDEI, 1983).

OD. rotundus pode gastarcerca de 40 minutos para escolher o local apropriado no corpo da vítima para morder (GREenHALL, 1971). Segundo SAZIMA (1978), os morcegos, ao terminarem de se alimentar, caminham um pouco sobre os animais antes de voarem. Ainda SAZIMA (1978), por algumas vezes, observou morcegos que predaram as partes baixas do animal, fazendo ataque terrestre, e saltitaram subindo no animal para se lançarem ao voo.

Três metodologias de controle de $D$. rotundus vêm sendo utilizadas em nosso País: uma delas é a aplicação da Warfarina Técnica sob a forma de pasta no dorso dos morcegos capturados em sua fonte de alimentação ou abrigos artificiais realizada pelo serviço oficial de defesa agropecuária; outra é a aplicação desta pasta ao redor dos ferimentos recentes provocados pelos quirópterosem animais de criação que pode ser executada pelos próprios criadores (GITTI, 2001). A terceira metodologia que, também pode ser utilizada pelos pecuaristas, é o uso da Warfarina em gel 
(Pour-on) no dorso dos animais agredidos (DELPIETRO et al., 1991; BRASIL, 2005).

O contato da saliva dos morcegos hematófagos com ferimento na pele pode permitir a passagem do vírus rábico à presa, caso o animal esteja infectado. Esse é o principal modo de transmissão da raiva, não sendo diferente do que acontece com a raiva transmitida por cães e gatos. Os morcegos hematófagos são os principais transmissores da raiva aos herbívoros domésticos (UIEDA et al., 2004).

O presente estudo tem por objetivo analisar a distribuição de ferimentos causados pelo $D$. rotundus em bovinos sob condições de campo.

\section{MATERIALEMÉTODOS}

As observações ocorreram no período de março 2005 a março de 2006, no Município de Valença, Estado do Rio de Janeiro, perfazendo um total de 580 animais examinados. Foram utilizadas 12 propriedades rurais que apresentavam sinais de ataque no rebanho, nas quais foram observados e analisados 101 bovinos que apresentavam ferimentos provocados por morcegos D. rotundus. Estes animais eram, em sua maioria, fêmeas em lactação e bovinos jovens lactentes de ambos os sexos, de diferentes cores de pelagem, com regime decriaçãosemi extensivo, sendo que animais jovens na sua maioria passavam a noite estabulados. Foram considerados animais estabulados todos aqueles que permaneciam a noite, ou parte dela, em ranchos e cercados ou bezerreiros onde o piso era de terra batida ou cimentado.

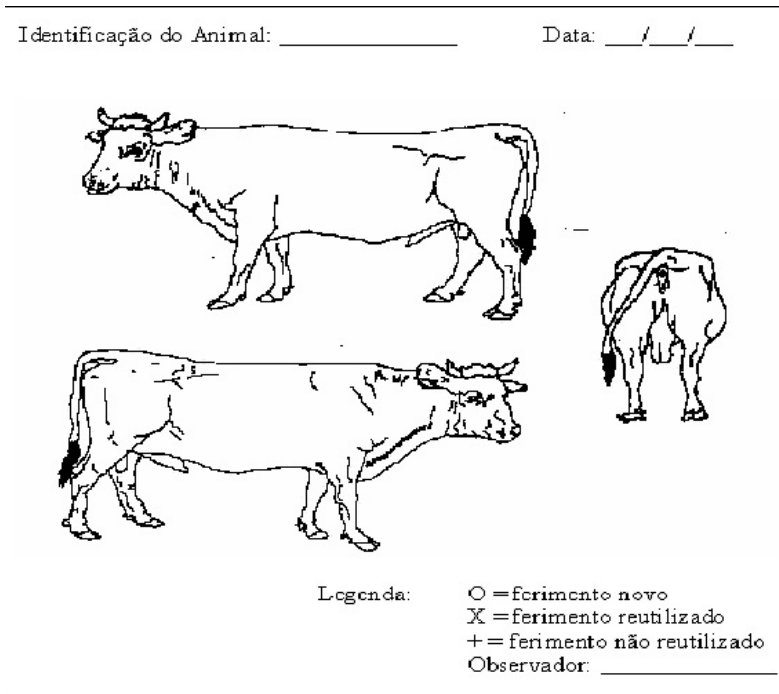

Fig. 1 -Modelo de ficha para anotações dos ferimentos efetuados pelomorcego D. rotundus (Fonte: GITTI, 2001)
Todos os bovinos foram conduzidos ao brete e/ ou examinados individualmente durante a ordenha para a observação de ferimentos causados pelos morcegos e os dados anotados em mapas corporais individuais (Fig. 1). Os animais que apresentaram ferimentos recentes, reutilizados ou cicatrizados, foram selecionados para avaliação dos resultados. Para a análise da região de ocorrência de ferimentos, o corpo dos bovinos foi dividido em quadrantes, onde os ferimentos foram anotados. O quadrante I representa a região ântero-superior, o quadrante II a região póstero-superior, o quadrante III a região ântero-inferior e o quadrante IV representa a região póstero-inferior do animal.

\section{RESULTADOS}

Na análise dos 580 mapas corporais (Fig. 1) dos animais observados, 101 bovinos apresentaram 276 ferimentos provocados por D. rotundus. Na distribuição dos ferimentos no corpo dos bovinos analisados foram observados 191 no primeiro quadrante, 46 no segundo, 20 no terceiro e 19 no quarto (Tabela 1). Por conseguinte, foram observados 237 ferimentos na metade superior perfazendo $85,87 \%$ do total e 39 na metade inferior do animal $(14,13 \%)$ como observado na Figura 2. A análise estatística para verificar significância da frequência de mordeduras nos quadrantes foi feita pelo "Teste $X^{2}$ " (Qui-Quadrado) no nível de $95 \%$ de significância. Confrontou-se um $X^{2} \mathrm{c}$ (Qui-quadrado calculado) de 287,41 com o tabelado $\left(X^{2} \mathrm{t}\right)$ de 7,815 para um grau de liberdade de valor igual a três. Desse modo, a frequência de mordeduras nos quadrantes difere significativamente nesse nível de significância. Ainda foram observados 211 ferimentos na metade anterior e 65 na metade posterior, resultando em $76,45 \%$ e $23,55 \%$, respectivamente (Fig. 2).

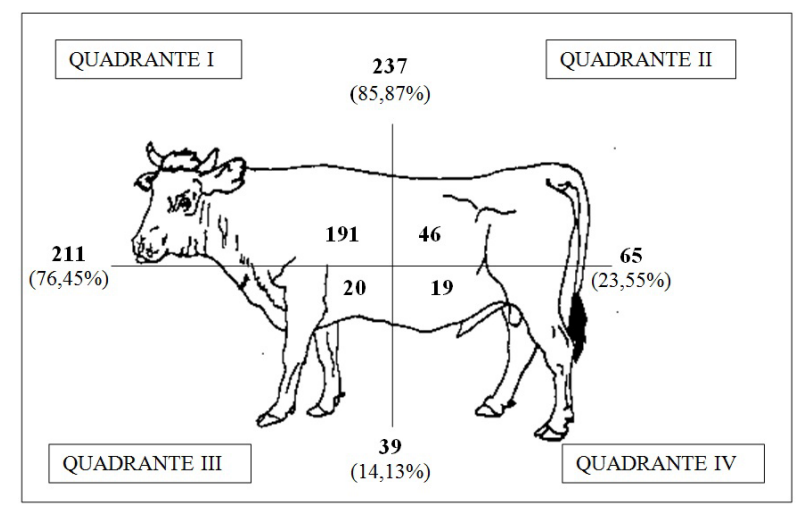

Fig. 2 - Distribuição dos ferimentos provocados por $D$. rotundus observados nos bovinos nas propriedades rurais, no Município de Valença, RJ, entre março de 2005 e março de 2006. Números centrais = quadrantes; Números periféricos $=$ metades. 
As propriedades que mais apresentaram animais com mordeduras na região ântero-superior foram: P2 (38), P11 (31) e P12 (31), como observado na Tabela 1. $\mathrm{NaP3}$ (5), naP5(3)enaP6(2), foi registradoumnúmero reduzido de mordeduras quando comparadas com as outras propriedades. As propriedades que apresentaram animais com maior número de ferimentos na região póstero-inferior foram: P2 e P11 com quatro ferimentos e P4 e P8 com três mordeduras.

Dos 580 animais pesquisados, 222 eram jovens em fase de amamentação representando $38,28 \%$ dos bovinos, sendoquedestes 159 estavamestabulados (71,62\%). Destaque para as propriedades P1, P7, P8, P9 eP11 em que o percentual de animais jovens estabulados é de $100 \%$. Não foram registrados animais estabulados na P3 e na P6 (Tabela 2). A avaliação da ocorrência de ferimentos em animais adultos e jovens foi feita pelo "Teste X" (Qui-Quadrado) no nível de 95\% de significância. Confrontou-se um $X^{2} \mathrm{c}$ (Qui-quadrado calculado $=31,89)$ corrigido pelo cálculo de Yates resultando em $X^{2} \mathrm{c}$ de 31,42 , com o tabelado $\left(X^{2} \mathrm{t}\right)$ de 3,841 para um grau de liberdade de valor igual a um. $O$ valor calculado é significativamente maior que o valor tabelado. Dessemodo, a frequência demordedurasem adultos diferesignificativamenteem relaçãoaos jovens nesse nível de significância.

Tabela 1 - Distribuição de ferimentos causados por morcego D. rotundus, de acordo com a região corporal dos bovinos, analisados em propriedades no Município de Valença, RJ, entre março de 2005 e março de 2006.

\begin{tabular}{cccccr}
\hline Propriedades $(\mathrm{P})$ & \multicolumn{4}{c}{ Região corporal } & Total de ferimentos \\
\cline { 2 - 5 } & $\begin{array}{c}\text { Antero } \\
\text { superior } \\
\left(1^{\circ} \mathrm{Q}\right)\end{array}$ & $\begin{array}{c}\text { Póstero } \\
\text { superior } \\
\left(2^{\circ} \mathrm{Q}\right)\end{array}$ & $\begin{array}{c}\text { Ântero } \\
\text { inferior } \\
\left(3^{\circ} \mathrm{Q}\right)\end{array}$ & $\begin{array}{c}\text { Póstero } \\
\text { inferior } \\
\left(4^{\circ} \mathrm{Q}\right)\end{array}$ & 21 \\
\hline 1 & 19 & 1 & - & 1 & 5 \\
2 & 38 & - & - & - & 55 \\
3 & 5 & 8 & 5 & 4 & 25 \\
4 & 14 & 6 & 2 & 3 & 4 \\
5 & 3 & 1 & - & - & 4 \\
6 & 2 & 1 & - & 1 & 10 \\
7 & 7 & 1 & 1 & 3 & 32 \\
8 & 21 & 6 & 2 & - & 14 \\
9 & 9 & 3 & 2 & - & 50 \\
11 & 11 & 1 & 2 & 2 & 50 \\
12 & 31 & 15 & - & 19 & 276 \\
\hline
\end{tabular}

(Quadrante $=$ Q).

Tabela 2 - Percentual de animais jovens estabulados pesquisados em propriedades no Município de Valença, RJ, entre março de 2005 e março de 2006.

\begin{tabular}{cccccr}
\hline $\begin{array}{c}\text { Propriedades } \\
(\mathrm{P})\end{array}$ & $\begin{array}{c}\text { Total } \\
\text { Observado }\end{array}$ & $\begin{array}{c}\text { Animais } \\
\text { jovens }\end{array}$ & $\begin{array}{c}\text { Percentual } \\
\text { de jovens }(\%)\end{array}$ & $\begin{array}{c}\text { Estabulados } \\
\text { Percentual de } \\
\text { estabulados }(\%)\end{array}$ \\
\hline 1 & 75 & 35 & 46,67 & 35 & $100,00 \%$ \\
2 & 70 & 20 & 28,57 & 11 & $55,00 \%$ \\
3 & 90 & 15 & 16,67 & - & $0,00 \%$ \\
4 & 51 & 20 & 39,22 & 6 & $35,00 \%$ \\
5 & 40 & 20 & 50,00 & - & $30,00 \%$ \\
6 & 13 & - & 0,00 & 7 & $0,00 \%$ \\
7 & 13 & 7 & 53,85 & 40 & $100,00 \%$ \\
8 & 80 & 40 & 50,00 & 13 & $100,00 \%$ \\
9 & 27 & 13 & 48,15 & 2 & $33,33 \%$ \\
10 & 18 & 6 & 33,33 & 15 & $100,00 \%$ \\
11 & 40 & 15 & 37,50 & 23 & $74,19 \%$ \\
\hline Total & 63 & 31 & 49,21 & 159 & $71,62 \%$ \\
\hline
\end{tabular}




\section{DISCUSSÃO}

A presença de ferimentos em várias regiões do corpo dos bovinos foi observada em estudo feito em cativeiro por PiccinIN et al.(1985), porém com predominância nos membros anteriores, dorso, membros posteriores e cernelha. Almeida et al. (2002) relataram que os ataques em animais de Curvelo e Codisburgo, no EstadodeMinas Gerais, ocorreram em várias partes do corpo dos bovinos, como coroa dos cascos, inserção da cauda, orelhas, focinho, dorso e região ínguino-crural e, com maior frequência, na região do pescoço. GITTI (2001) levantou a hipótese de que os morcegos $D$. rotundus podem ter hábitos comportamentais um pouco diferentes em relação à preferência anatômica para espoliarosbovinos, dependendodalocalidadeem que seencontram. Como as fêmeas ensinam aos seus filhotes quais os tipos de presas que eles devem atacar (SChMidT et al., 1980; Greenhall et al., 1983; FenTON, 1975), provavelmente as regiões anatômicas também são passadas de geração a geração com possibilidades de, em determinadas localidades geográficas, haver divergência nos números de mordeduras, conforme a localização anatômica nos bovinos.

Por meio da análise do número de mordeduras por quadrantes, pôde-se constatar um elevado número de mordedurasna metadesuperior $(85,87 \%)$,enquantoque apenas $14,13 \%$ foram observados na metade inferior do corpo. SAZIMA (1978) relatou, em uma propriedade no MunicípiodeCampinas,EstadodeSãoPaulo, a ocorrência de $84,74 \%$ e $15,26 \%$ de mordeduras na parte superior einferior dosbovinos, respectivamente. Estes resultados se assemelham ao do presente estudo, onde a grande maioria dos ferimentos ocorreu na parte superior dos animais e o primeiro quadrante com resultados mais significativos. Resultados semelhantes foram obtidos por Gптт (2001) que em trabalho realizado em seis propriedades rurais com bovinos, foi encontrado $92,5 \%$ de ferimentosnametadesuperiordosbovinospesquisados.

O local onde os animais passam a noite pode interferir no número de mordeduras na metade superior, quando comparada com a inferior. Os animais estabulados têm maischances deapresentar ferimentos nas regiões corporais baixas, tais como, pernas, peito, úbereetestículos, eosquepassama noiteno pasto mais chances de aparecerem com ferimentos na metade superior, pois o capim serve como barreira física para os morcegos, atrapalhando o seu pouso esua caminhada até o animal (SAZIMA, 1978; GITTI, 2001).

O fato de ter sido observado um número maior de ferimentos na metade inferior do corpo nesta pesquisa $(14,13 \%)$, comparadoaotrabalho de GiтTI (2001)em que foiobservado7,45\%, provavelmentesejajustificadopor ser uma criação com um regime semi extensivo, onde a maioria dos bovinos jovens permanecia estabulada durante a noite $(71,62 \%)$ ao contrário do trabalho do autor anterior em que todos os animais pesquisados possuíam diferentes características comportamentais, temperamentaise demanejo eque passavam a noiteno pasto, ea pesquisa foi feita em diferentes municípios do Estado do Rio de Janeiro. TuRNER (1975) citou que $D$. rotundus geralmente escolhe animais de um temperamento mais dócil, e UIEDA (1982) relatou que um fator importante na seleção das presas é a acessibilidade confirmando os resultados deste estudo.

Oelevadonúmero de mordeduras encontradas na partesuperior do corpo dosbovinos (85,87\%) confirma a justificativa de se utilizar novas metodologias de controle da população de morcegos hematófagos $D$. rotundus, valorizandoocontatodestemorcegonaquela região corporal. Produtos vampiricidas de aplicação dorsal em bovinos podem ser uma boa opção para seu controle, contudo, há a necessidade da execução de mais estudos científicos que possam comprovar ou refutar sua eficácia, visto queexistem produtos comerciais quesão aplicados na região superior dos bovinos, mas sem publicações científicas da metodologia e dos resultados que certifiquem sua eficácia.

\section{CONCLUSÕES}

Levando-se em consideração o objetivo proposto e a metodologia utilizada, conclui-se que:

O D. rotundus utiliza diferentes locais para se alimentar nos bovinos e a região corporal destes animais que apresentou maior número de ferimentos provocados por este morcego foi a ântero superior.

O elevado número de ferimentos encontrados na metade superior dos bovinos $(85,87 \%)$ justifica a adoção de metodologias de controle que se utilizem do contato corporal dos morcegos no dorso do animal para promover sua ação.

\section{REFERÊNCIAS}

ALENCAR, O.A.;SILVA, G.A.P.; ARRUDA, M.M.;SOARES, A.J.; GUERRA, D.Q. Aspectos biológicos de Desmodus rotundus rotundus (Chiroptera) no nordeste do Brasil. Pesquisa Veterinária Brasileira, v.14, n.4, p.95-103, 1994.

ALMEIDA, E.O.; MOREIRA, E.C.; NAVEDA, L.A.B.; HERRMANN, G.P. Combate ao Desmodus rotundus rotundus (E. Geoffroy, 1810) na região cárstica de Cordisburgo e Curvelo, Minas Gerais. Arquivo Brasileiro de Medicina Veterinária e Zootecnia, v.54, n.2, p.1-13, 2002.

BRASIL. Ministério da Agricultura, Pecuária e Abastecimento. Controle da raiva dos herbívoros. Brasília: MAPA, 2005. 104p. (Manual Técnico).

CRESPO, J.A.; FERNANDEZ, S.S.; BURNS, R.J.; MITCHELL, G.C. Observaciones sobre el 
comportamiento del vampiro común (Desmodus rotundus) al alimentarse en condiciones naturales. Tecnica Pecuária Mexicana, n. 27, p.39-45, 1971.

DELPIETRO, H.A.; RUSSO, G.; ALLI, C.; PATIRE, J. Una nueva forma de combatir vampiros. Veterinária Argentina, v.77, n.7, p.455-463, 1991.

FENTON, M.B. Bats. New York: Facts on file, 1975. 207p.

FUNDAÇÃO NACIONAL DE SAÚDE. Brasil. Morcegos em áreas urbanas e rurais: Manual de manejo e controle. Brasília: Fundação Nacional de Saúde, 1998. 117p.

GITTI, C.B. Controle do morcego hematófago Desmodus rotundus (Geoffroy, 1810) pela aspersão de Warfarina em ovinos e bovinos. 2001. 89p. Tese (Doutorado em Ciências Veterinárias) - Universidade Federal Rural do Rio de Janeiro, Rio de Janeiro, 2001.

GREENHALL, A.M. Use of mist nets and strychnine for vampire bat control in Trinidad. Journal of Mammalogy, v. 44, n.3, p.396-399, 1963.

GREENHALL, A.M.;SCHMIDT,U.;LÓPEZ-FORMENT,W. Field observations on the mode of attack of the vampire bat (D. rotundus) in Mexico. Anais do Instituto de Biologia da Universidad Autónoma de Mexico, v.40, n.2, p.242-245, 1969.

GREENHALL, A.M. The use of a precipitin test to determine host preferences of the vampire bats, $D$. rotundus and Diaemus youngii. Bijdragen Dierkunde, v.40, p.36-39, 1970.

GREENHALL, A.M. Lucha contra los murciélagos vampiros. Boletin de la Oficina Sanitária Panamericana, v.71, n.3, p.231-245, 1971.

GREENHALL, A.M.; JOERMANN, G.; SCHMIDT, U. Mammalian Species. Desmodus rotundus. American Society of Mammalogy, n. 202, p.1-6, 1983.

MALAGA-ALBA, A. Vampire bats as a carrier of rabies. American Journal of Public Health, n.44, p.909-918, 1954.

PICCININI, R.S.; PERACCHI, A.L.; SOUZA, J.C.P.; ALBUQUERQUE, S.T.; RAIMUNDO, S.D.L.; TANNURE,
A.M.; FURTADO, L.L. Comportamento do morcego hematófago Desmodus rotundus (Chiroptera) relacionado com a taxa de ataque a bovinos em cativeiro. Pesquisa Veterinária Brasileira, v.5, n.4, p.11-116, 1985.

SAZIMA, I. Aspectos do comportamento alimentar do morcego hematófago Desmodus rotundus. Boletim de Zoologia da Universidade de São Paulo, n.3, p.97-120, 1978.

SAZIMA, I.; UIEDA, W. Feeding behavior of the whitewinged vampire bat Diaemus youngii, on poultry. Journal of Mammalogy, v.61, n.1, p.102-104, 1980.

SCHMIDT, C.; SCHMIDT, U.; MANSKE, U. Observations of the behavior of Orphaned juveniles in the common vampire bat (Demodus rotundus). In: INTERNATIONAL BAT RESEARCH CONFERENCE, 1980, Texas, USA. Proceedings. Texas: Tech Press, Lubbuck, 1980. 434p.

TADDEI, V.A. Morcegos: algumas considerações sistemáticas e biológicas. Boletim Técnico da CATI, n.172, p.1-31, 1983.

TURNER, D.C. The vampire bat - A field study in behaviour and ecology. Baltimore and London: Johns Hopkins Univ. Press, 1975. 145p.

UIEDA, W. Aspectos do comportamento alimentar das três espécies de morcegos hematófagos (Chiroptera, Phyllostomidae). 1982. 166f. Dissertação (Mestrado em Ecologia) - Instituto de Biologia, Universidade Estadual de Campinas, Campinas, 1982.

UIEDA, W. Morcegos hematófagos e a raiva dos herbívoros no Brasil. In: SEMINÁRIO DE CIÊNCIAS DA FACULDADES INTEGRADAS DE UBERABA, 1. 1994, Uberaba, MG. Resumos. Uberaba: 1987. p.13-29.

UIEDA, W.; CARDOSO, M.; ALVES, G.M. Fauna de morcegos da região de Botucatu. In: UIEDA W.; PALEARI L.M. (Ed.). Flora e fauna: um dossiê ambiental. São Paulo: Ed.Unesp. 2004. p.99-119.

Recebido em 14/11/08

Aceito em 4/6/10 